\title{
ON AN INEQUALITY OF DIANANDA. PART II.
}

PENG GAO

Received 31 October 2004 and in revised form 17 March 2005

We extend the result in part I, 2003, of certain inequalities among the generalized power means.

\section{Introduction}

Let $P_{n, r}(\mathbf{x})$ be the generalized weighted means: $P_{n, r}(\mathbf{x})=\left(\sum_{i=1}^{n} q_{i} x_{i}^{r}\right)^{1 / r}$, where $P_{n, 0}(\mathbf{x})$ denotes the limit of $P_{n, r}(\mathbf{x})$ as $r \rightarrow 0^{+}, \mathbf{x}=\left(x_{1}, x_{2}, \ldots, x_{n}\right)$ and $q_{i}>0(1 \leq i \leq n)$ are positive real numbers with $\sum_{i=1}^{n} q_{i}=1$. In this paper, we let $q=\min q_{i}$ and always assume $n \geq 2$, $0 \leq x_{1}<x_{2}<\cdots<x_{n}$.

We define $A_{n}(\mathbf{x})=P_{n, 1}(\mathbf{x}), G_{n}(\mathbf{x})=P_{n, 0}(\mathbf{x}), H_{n}(\mathbf{x})=P_{n,-1}(\mathbf{x})$, and we will write $P_{n, r}$ for $P_{n, r}(\mathbf{x}), A_{n}$ for $A_{n}(\mathbf{x})$, and similarly for other means when there is no risk of confusion.

For mutually distinct numbers $r, s, t$ and any real numbers $\alpha$, $\beta$, we define

$$
\Delta_{r, s, t, \alpha, \beta}=\left|\frac{P_{n, r}^{\alpha}-P_{n, t}^{\alpha}}{P_{n, r}^{\beta}-P_{n, s}^{\beta}}\right|,
$$

where we interpret $P_{n, r}^{0}-P_{n, s}^{0}$ as $\ln P_{n, r}-\ln P_{n, s}$. When $\alpha=\beta$, we define $\Delta_{r, s, t, \alpha}$ to be $\Delta_{r, s, t, \alpha, \alpha}$. We also define $\Delta_{r, s, t}$ to be $\Delta_{r, s, t, 1}$.

Bounds for $\Delta_{r, s, t, \alpha, \beta}$ have been studied by many mathematicians. For the case $\alpha \neq \beta$, we refer the reader to the articles $[2,5,10]$ for the detailed discussions. In the case $\alpha=\beta$ and $r>s>t$, we seek the bound

$$
f_{r, s, t, \alpha}(q) \geq \Delta_{r, s, t, \alpha}
$$

and the bound

$$
\Delta_{r, s, t, \alpha} \geq g_{r, s, t, \alpha}(q),
$$

where $f_{r, s, t, \alpha}(q)$ is a decreasing function of $q$ and $g_{r, s, t, \alpha}(q)$ is an increasing function of $q$.

For $r=1, s=0, \alpha=0, t=-1$, in (1.2) and (1.3), we can take $f_{1,0, t, 0}(q)=1 / q, g_{1,0, t, 0}(q)=$ $1 /(1-q)$. When $q_{i}=1 / n, 1 \leq i \leq n$, these are the well-known Sierpinski's inequalities [12] (see [6] for a refinement of this). If we further require $t, \alpha>0$, then consideration of 
the case $n=2, x_{1} \rightarrow 0, x_{2}=1$ leads to the choice $f_{r, s, t, \alpha}=C_{r, s, t}\left((1-q)^{\alpha}\right), g_{r, s, t, \alpha}=C_{r, s, t}\left(q^{\alpha}\right)$, where

$$
C_{r, s, t}(x)=\frac{1-x^{1 / t-1 / r}}{1-x^{1 / s-1 / r}}, \quad t>0 ; \quad C_{r, s, 0}(x)=\frac{1}{1-x^{1 / s-1 / r}} .
$$

We will show in Lemma 2.1 that $C_{r, s, t}(x)$ is an increasing function of $x(0<x<1)$, so the above choice for $f, g$ is plausible. From now on, we will assume $f, g$ to be so chosen.

Note when $t>0$, the limiting case $\alpha \rightarrow 0$ in (1.2) leads to Liapunov's inequality (see [8, page 27]):

$$
\Delta_{r, s, t, 0}=\frac{\ln P_{n, r}-\ln P_{n, t}}{\ln P_{n, r}-\ln P_{n, s}} \leq \frac{s(r-t)}{t(r-s)}=: C(r, s, t) .
$$

From this (or by letting $q \rightarrow 0$ when $\alpha=1$ ), one easily deduces the following result of Hsu [9] (see also [1]): $\Delta_{r, s, t} \leq C(r, s, t), r>s>t>0$.

For $n=2$ and $r>s>t \geq 0, \Delta_{r, s, t, \alpha} \rightarrow(r-t) /(r-s)$ as $x_{2} \rightarrow x_{1}$. Therefore, the two inequalities (1.2) and (1.3) cannot hold simultaneously in general. Now for any set $\{a, b, c\}$ with $a, b, c$ mutually distinct and nonnegative, we let $r=\max \{a, b, c\}, t=\min \{a, b, c\}$, $s=\{a, b, c\} \backslash\{r, t\}$. By saying (1.2) (resp. (1.3)) holds for the set $\{a, b, c\}, \alpha>0$, we mean (1.2) (resp. (1.3)) holds for $r>s>t \geq 0, \alpha>0$.

In the case $\alpha=1$, a result of Diananda (see [3, 4]) (see also [1, 11]) shows that (1.2) and (1.3) hold for $\{1,1 / 2,0\}$ and his result has recently been extended by the author [7] to the cases $\{r, 1,0\}$ and $\{r, 1,1 / 2\}$ with $r \in(0, \infty)$. It is the goal of this paper to further extend the results in [7].

\section{Lemmas}

LeMmA 2.1. For $0<x<1,0 \leq t<s<r, C_{r, s, t}(x)$ is a strictly increasing function of $x$. In particular, for $0<q \leq 1 / 2, C_{r, s, t}(1-q) \geq C_{r, s, t}(q)$.

Proof. We may assume $t>0$. Note $C_{r, s, t}(x)=C_{1, s / r, t / r}\left(x^{1 / r}\right)$, thus it suffices to prove the lemma for $C_{1, r, s}$ with $1>r>s>0$. By the Cauchy mean value theorem,

$$
\frac{1 / s-1}{1 / r-1} \cdot \frac{1-x^{1 / r-1}}{1-x^{1 / s-1}}=\eta^{1 / r-1 / s}<x^{1 / r-1 / s}
$$

for some $x<\eta<1$ and this implies $C_{1, r, s}^{\prime}(x)>0$ which completes the proof.

Lemma 2.2. For $1 / 2<r<1, C_{1, r, 1-r}(1 / 2)>r /(1-r)$.

Proof. By setting $x=r /(1-r)>1$, it suffices to show $f(x)>0$ for $x>1$, where $f(x)=1-$ $2^{-x}-x\left(1-2^{-1 / x}\right)$. Now $f^{\prime \prime}(x)=(\ln 2)^{2} 2^{-x} x^{-3}\left(2^{x-1 / x}-x^{3}\right)$ and let $g(x)=(x-1 / x) \ln 2-$ $3 \ln x$. Note $g^{\prime}(x)$ has one root in $(1, \infty)$ and $g(1)=0$, it follows that $g(x)$, hence $f^{\prime \prime}(x)$, has only one root $x_{0}$ in $(1, \infty)$. Note when $f^{\prime \prime}(x)>0$ for $x>x_{0}$, this together with the observation that $f(1)=0, f^{\prime}(1)=\ln 2-1 / 2>0, \lim _{x \rightarrow \infty} f(x)=1-\ln 2>0$ shows $f(x)>$ 0 for $x>1$.

LEMMA 2.3. Let $0<q \leq 1 / 2$. For $0<s<r<1, r+s \geq 1, C_{1, r, s}(1-q)>(1-s) /(1-r)$. For $0 \leq s<1<r, C_{r, 1, s}(1-q)>(r-s) /(r-1)$ and for $1<s<r, C_{r, s, 1}(1-q)>(r-1) /(r-s)$. 
Proof. We will give a proof for the case $1>r>s>0, r+s \geq 1$ here and the proofs for the other cases are similar. We note first that in this case $1 / 2<r<1$. By Lemma 2.1, it suffices to prove $C_{1, r, s}(1 / 2)>(1-s) /(1-r)$. Consider

$$
f(s)=(1-r)\left(1-\left(\frac{1}{2}\right)^{1 / s-1}\right)-(1-s)\left(1-\left(\frac{1}{2}\right)^{1 / r-1}\right) .
$$

We have $f(r)=0$ and Lemma 2.2 implies $f(1-r)>0$. Now $f^{\prime}(r)=2^{1-1 / r} g(1 / r)$, where $g(x)=-\ln 2\left(x^{2}-x\right)+2^{x-1}-1$ with $1<x<2$. One checks easily $g(1)=g^{\prime}(1)=0, g^{\prime \prime}(x)<$ 0 which implies $g(x)<0$. Hence, $f^{\prime}(r)<0$, this combined with the observation that

$$
f^{\prime \prime}(s)=(1-r) \ln 2\left(\frac{1}{2}\right)^{1 / s-1} \frac{(2 s-\ln 2)}{s^{4}}
$$

has at most one root and $f^{\prime \prime}(r)>0, f(1-r)>0, f(r)=0$ imply that $f(s)>0$ for $1-r \leq$ $s<r$.

\section{The main theorems}

Theorem 3.1. Let $\alpha=1$. Inequality (1.2) holds for the set $\{1, r, s\}$, with $1, r, s$ mutually distinct and $r>s \geq 0, r+s \geq 1$. The equality holds if and only if $n=2, x_{1}=0, q_{1}=q$.

Proof. The case $s=0$ was treated in [7], so we may assume $s>0$ here. We will give a proof for the case $1>r>s>0$ here and the proofs for the other cases are similar. Define

$$
D_{n}(\mathbf{x})=A_{n}-P_{n, r}-C(1-q)\left(A_{n}-P_{n, s}\right), \quad C(x)=\frac{1-x^{1 / r-1}}{1-x^{1 / s-1}} .
$$

By Lemma 2.3, we need to show $D_{n} \geq 0$ and we have

$$
\frac{1}{q_{n}} \frac{\partial D_{n}}{\partial x_{n}}=1-P_{n, r}^{1-r} x_{n}^{r-1}-C(1-q)\left(1-P_{n, s}^{1-s} x_{n}^{s-1}\right)
$$

By a change of variables: $x_{i} / x_{n} \rightarrow x_{i}, 1 \leq i \leq n$, we may assume $0 \leq x_{1}<x_{2}<\cdots<x_{n}=$ 1 in (3.2) and rewrite it as

$$
g_{n}\left(x_{1}, \ldots, x_{n-1}\right):=1-P_{n, r}^{1-r}-C(1-q)\left(1-P_{n, s}^{1-s}\right)
$$

We want to show $g_{n} \geq 0$. Let $\mathbf{a}=\left(a_{1}, \ldots, a_{n-1}\right) \in[0,1]^{n-1}$ be the point in which the absolute minimum of $g_{n}$ is reached. We may assume $a_{1} \leq a_{2} \leq \cdots \leq a_{n-1}$. If $a_{i}=a_{i+1}$ for some $1 \leq i \leq n-2$ or $a_{n-1}=1$, by combing $a_{i}$ with $a_{i+1}$ and $q_{i}$ with $q_{i+1}$, or $a_{n-1}$ with 1 and $q_{n-1}$ with $q_{n}$, it follows from Lemma 2.1 that we can reduce the determination of the absolute minimum of $g_{n}$ to that of $g_{n-1}$ with different weights. Thus without loss of generality, we may assume $a_{1}<a_{2}<\cdots<a_{n-1}<1$.

If $\mathbf{a}$ is a boundary point of $[0,1]^{n-1}$, then $a_{1}=0$, and we can regard $g_{n}$ as a function of $a_{2}, \ldots, a_{n-1}$, then we obtain

$$
\nabla g_{n}\left(a_{2}, \ldots, a_{n-1}\right)=0
$$


1384 On an inequality of Diananda. Part II.

Otherwise $a_{1}>0$, a is an interior point of $[0,1]^{n-1}$ and

$$
\nabla g_{n}\left(a_{1}, \ldots, a_{n-1}\right)=0
$$

In either case $a_{2}, \ldots, a_{n-1}$ solve the equation

$$
(r-1) P_{n, r}^{1-2 r} x^{r-1}+C(1-q)(1-s) P_{n, s}^{1-2 s} x^{s-1}=0 .
$$

The above equation has at most one root (regarding $P_{n, r}, P_{n, s}$ as constants), so we only need to show $g_{n} \geq 0$ for the case $n=3$ with $0=a_{1}<a_{2}=x<a_{3}=1$ in (3.3). In this case we regard $g_{3}$ as a function of $x$ and we get

$$
\frac{1}{q_{2}} g_{3}^{\prime}(x)=P_{3, r}^{1-2 r} x^{r-1} h(x)
$$

where

$$
h(x)=r-1+(1-s) C(1-q)\left(q_{2} x^{s / 2}+q_{3} x^{-s / 2}\right)^{(1-2 s) / s}\left(q_{2} x^{r / 2}+q_{3} x^{-r / 2}\right)^{(2 r-1) / r} .
$$

If $q_{2}=0$ (note $\left.q_{3}>0\right)$, then

$$
h(x)=r-1+(1-s) C(1-q) q_{3}^{1 / s-1 / r} x^{s-r} .
$$

One easily checks that in this case $h(x)$ has exactly one root in $(0,1)$. Now assume $q_{2}>0$, then

$$
h^{\prime}(x)=(1-s) C(1-q) P_{3, s}^{1-3 s} P_{3, r}^{r-1} x^{-(r+s+2) / 2} p(x)
$$

where

$$
p(x)=(r-s)\left(q_{2}^{2} x^{r+s}-q_{3}^{2}\right)+(r+s-1) q_{2} q_{3}\left(x^{r}-x^{s}\right) .
$$

Now

$$
p^{\prime}(x)=x^{s-1}\left(\left(r^{2}-s^{2}\right) q_{2}^{2} x^{r}+(r+s-1) q_{2} q_{3}\left(r x^{r-s}-s\right)\right):=x^{s-1} q(x) .
$$

If $r+s \geq 1$, then $q^{\prime}(x)>0$ which implies there can be at most one root for $p^{\prime}(x)=0$. Since $p(0)<0$ and $\lim _{x \rightarrow \infty} p(x)=+\infty$, we conclude that $p(x)$, hence $h^{\prime}(x)$, has at most one root. Since $h(1)<0$ by Lemma 2.3 and $\lim _{x \rightarrow 0^{+}} h(x)=+\infty$, this implies $h(x)$ has exactly one root in $(0,1)$.

Thus $g_{3}^{\prime}(x)$ has only one root $x_{0}$ in $(0,1)$. Since $g_{3}^{\prime}(1)<0, g_{3}(x)$ takes its maximum value at $x_{0}$. Thus $g_{3}(x) \geq \min \left\{g_{3}(0), g_{3}(1)\right\}=0$. 
Thus we have shown $g_{n} \geq 0$, hence $\partial D_{n} / \partial x_{n} \geq 0$ with equality holding if and only if $n=1$ or $n=2, x_{1}=0, q_{1}=q$. By letting $x_{n}$ tend to $x_{n-1}$, we have $D_{n} \geq D_{n-1}$ (with weights $\left.q_{1}, \ldots, q_{n-2}, q_{n-1}+q_{n}\right)$. Since $C$ is an increasing function of $q$, it follows by induction that $D_{n}>D_{n-1}>\cdots>D_{2}=0$ when $x_{1}=0, q_{1}=q$ in $D_{2}$. Else $D_{n}>D_{n-1}>\cdots>D_{1}=0$. Since we assume $n \geq 2$ in this paper, this completes the proof.

The relations between (1.2) and (1.5) seem to suggest that if (1.2) holds for $r>s>$ $t \geq 0, \alpha>0$, then (1.2) also holds for $r>s>t \geq 0, k \alpha$ with $k<1$ and if (1.3) holds for $r>s>t \geq 0, \alpha>0$, then (1.3) also holds for $r>s>t \geq 0, k \alpha$ with $k>1$. We do not know the answer in general but for a special case, we have the following.

Theorem 3.2. Let $r>s>0$. If (1.2) holds for $\{r, s, 0\}, \alpha>0$, then it also holds for $\{r, s, 0\}, k \alpha$ with $k>1$. If (1.3) holds for $\{r, s, 0\}, \alpha>0$, then it also holds for $\{r, s, 0\}, k \alpha$ with $0<k<1$.

Proof. We will only prove the first assertion here and the second can be proved similarly. By the assumption, we have

$$
P_{n, r}^{\alpha}-G_{n}^{\alpha} \geq \frac{1}{1-\left(q^{\alpha}\right)^{1 / s-1 / r}}\left(P_{n, r}^{\alpha}-P_{n, s}^{\alpha}\right)
$$

We write the above as

$$
P_{n, s}^{\alpha} \geq\left(q^{\alpha}\right)^{1 / s-1 / r} P_{n, r}^{\alpha}+\left(1-\left(q^{\alpha}\right)^{1 / s-1 / r}\right) G_{n}^{\alpha} .
$$

We now need to show for $k>1$,

$$
P_{n, s}^{k \alpha} \geq\left(q^{k \alpha}\right)^{1 / s-1 / r} P_{n, r}^{k \alpha}+\left(1-\left(q^{k \alpha}\right)^{1 / s-1 / r}\right) G_{n}^{k \alpha} .
$$

Note by (3.14), via setting $w=\left(q^{k \alpha}\right)^{1 / s-1 / r}, x=G_{n} / P_{n, r}$, it suffices to show

$$
f(x)=:\left(w+(1-w) x^{k}\right)^{1 / k}-w^{1 / k}-\left(1-w^{1 / k}\right) x \leq 0,
$$

for $0 \leq w, x \leq 1$. Note

$$
f^{\prime}(x)=(1-w)\left(w x^{-k}+(1-w)\right)^{1 / k-1}-\left(1-w^{1 / k}\right)
$$

thus $f^{\prime}(x)$ can have at most one root in $(0,1)$, note also $f(0)=f(1)=0$ and $f^{\prime}(1)>0$, we then conclude $f(x) \leq 0$ for $0 \leq x \leq 1$ and this completes the proof.

\section{Acknowledgment}

The author is grateful to the referees for their valuable comments and suggestions. 


\section{On an inequality of Diananda. Part II.}

\section{References}

[1] P. S. Bullen, D. S. Mitrinović, and P. M. Vasić, Means and Their Inequalities, Mathematics and Its Applications (East European Series), vol. 31, D. Reidel Publishing, Dordrecht, 1988.

[2] D. I. Cartwright and M. J. Field, A refinement of the arithmetic mean-geometric mean inequality, Proc. Amer. Math. Soc. 71 (1978), no. 1, 36-38.

[3] P. H. Diananda, On some inequalities of H. Kober, Proc. Cambridge Philos. Soc. 59 (1963), 341-346.

[4] - "On some inequalities of H. Kober": Addendum, Proc. Cambridge Philos. Soc. 59 (1963), 837-839.

[5] P. Gao, Certain bounds for the difference of means, JIPAM. J. Inequal. Pure Appl. Math. 4 (2003), no. 4 , article $76,10 \mathrm{pp}$.

[6] _ Ky Fan inequality and bounds for differences of means, Int. J. Math. Math. Sci. 2003 (2003), no. 16, 995-1002.

[7] _ On an inequality of Diananda, Int. J. Math. Math. Sci. 2003 (2003), no. 32, 20612068.

[8] G. H. Hardy, J. E. Littlewood, and G. Pólya, Inequalities, Cambridge University Press, Cambridge, 1952.

[9] L. C. Hsu, Questions 1843,1844, Math. Student 23 (1955), 121.

[10] A. McD. Mercer, Some new inequalities involving elementary mean values, J. Math. Anal. Appl. 229 (1999), no. 2, 677-681.

[11] D. S. Mitrinović, J. E. Pečarić, and A. M. Fink, Classical and new inequalities in analysis, Mathematics and Its Applications (East European Series), vol. 61, Kluwer Academic, Dordrecht, 1993.

[12] W. Sierpiński, On an inequality for arithmetic, geometric and harmonic means, Warsch. Sitzungsber. 2 (1909), 354-358 (Polish).

Peng Gao: Department of Mathematics, University of Michigan, Ann Arbor, MI 48109, USA

E-mail address: penggao@umich.edu 


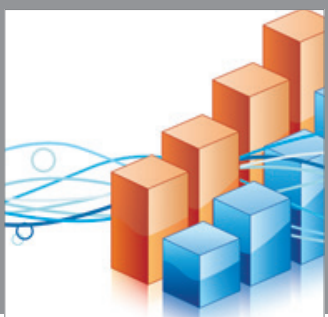

Advances in

Operations Research

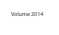

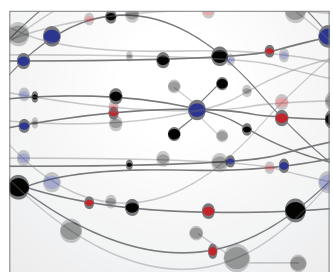

\section{The Scientific} World Journal
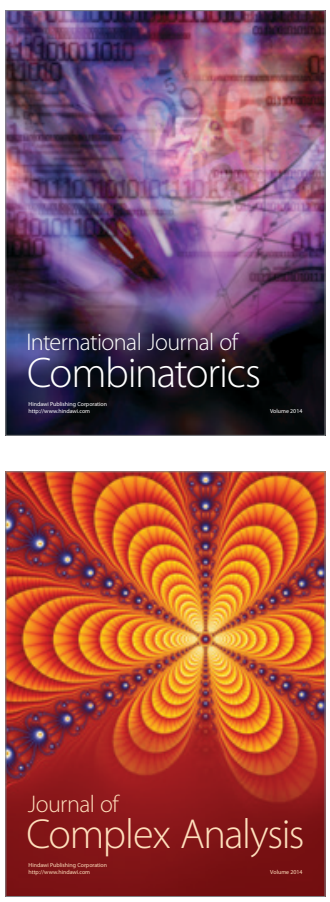

International Journal of

Mathematics and

Mathematical

Sciences
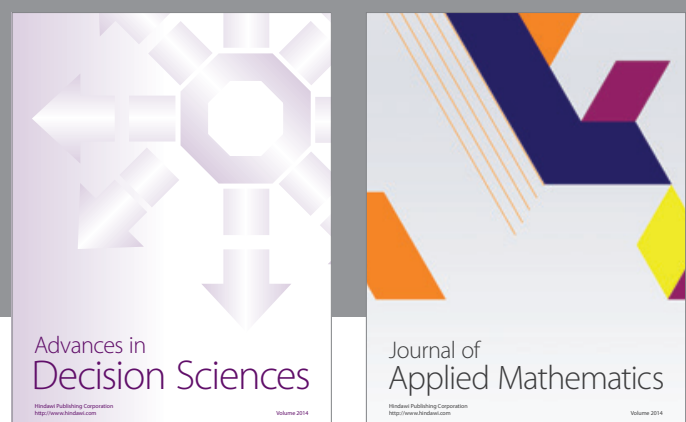

Journal of

Applied Mathematics
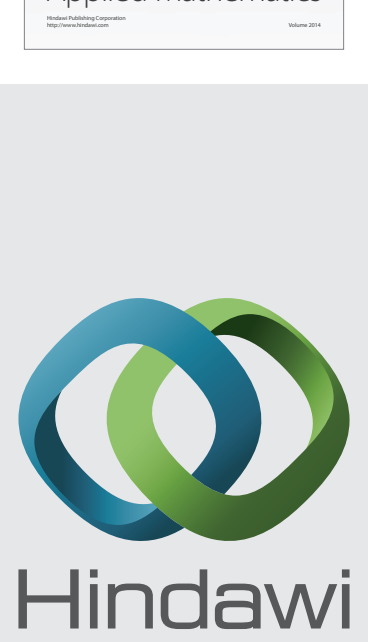

Submit your manuscripts at http://www.hindawi.com
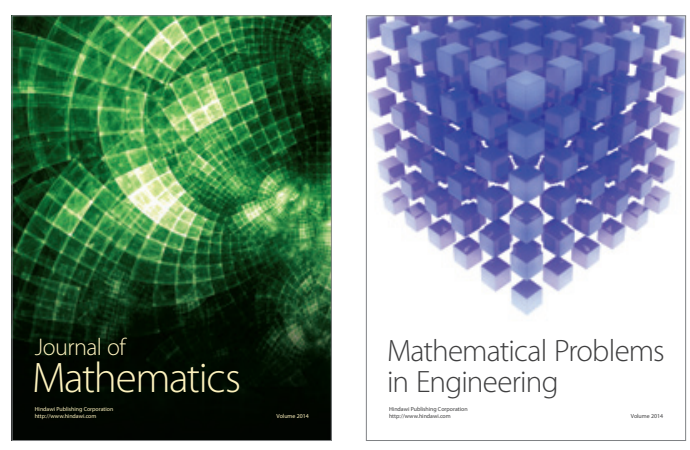

Mathematical Problems in Engineering
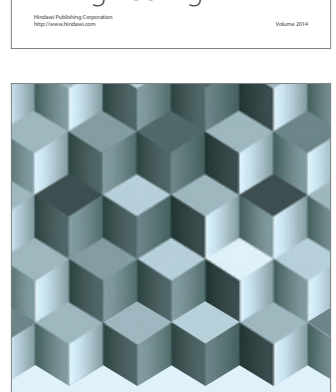

Journal of

Function Spaces
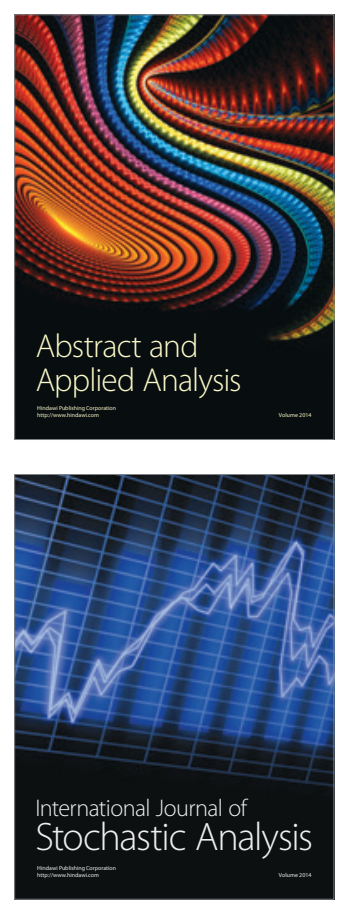

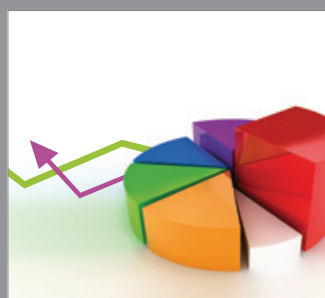

ournal of

Probability and Statistics

Promensencen
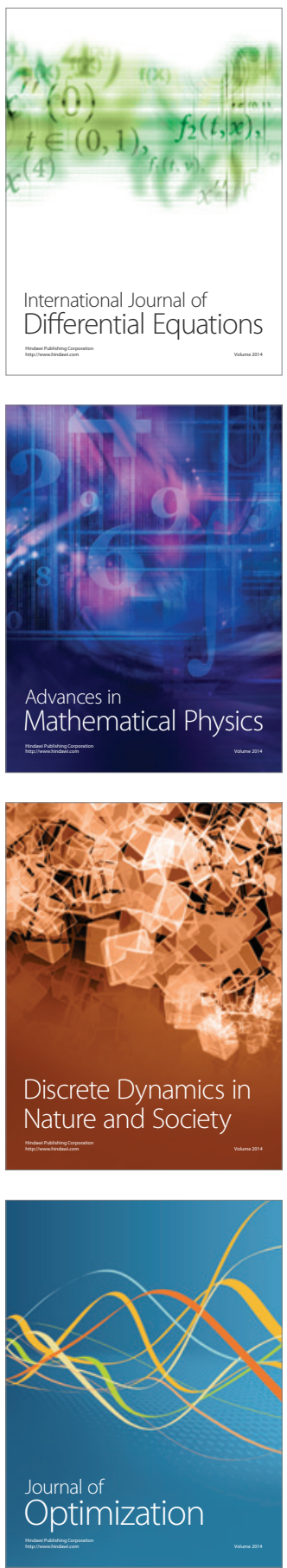